\title{
Simulation of Touch Sensor Switch Control Circuit
}

\author{
Mingyu Fan, ${ }^{\mathrm{a}, \mathrm{b},}$, Daisuke Tanaka ${ }^{\mathrm{a}}$, Akira Yamawaki ${ }^{\mathrm{a}}$, Seiichi Serikawa ${ }^{\mathrm{a}}$ \\ ${ }^{\text {a }}$ Kyushu Institute of Technology \\ 1-1, Sensui, Tobata, Kitakyushu, Japan 804-8550 \\ ${ }^{\mathrm{b}}$ Yangzhou University
}

196, Huayangxilu, Yangzhou, Jiangsu, China 225-127

*Corresponding Author: youranzhiyue @ 163.com

\begin{abstract}
A lot of push-button and touch panel is appeared to control almost all equipments. However, there are still some drawbacks. This paper focuses on simulation a kind of touch switch circuit which using metal film as a switch. The simulation is based on PSPICE software which simulates the action of finger touch by wave form. The simulation result shows that this circuit can be used as a switch and the accuracy is very high. This switch gets rid of the traditional shape of the general switches, easy to use, has wide application and a great prospect.
\end{abstract}

Keywords: touch switch, high accuracy, arbitrary shape.

\section{Introduction}

Traditional switch has two mechanical contacts ${ }^{(1)}$. The contacts have to be connected then electric current can flow between them and form a loop that means the switch is "closed". Most of the mechanical switch will produce a noise, furthermore, it is limited by low reliability, high failure rate and easy to wear so that can't satisfy the development of technology and application requirements.

Touch switch generally refers to a new type of switch manually controlled by tapping it. When finger touch the switch, human body is act as a kind of conductor. There are three types of touch switches: capacitance touch switch, resistance touch switch and piezo touch switch ${ }^{(2)}$. The touch panel switch proposed by Professor Serikawa ${ }^{(3)}$ has the function of bending and addition. Its power consumption is very low as if it isn't touched no power will product. However, the accuracy of this switch is not high and it will be easily affected by environment noisy. The number of added switch has a limitation. Just like their switch, the shape of touch switch can be cut to arbitrary shape too. It can be stuck on any convenient places. Add more switches are alterable in this circuit but the process circuit should be added at the same time. The switch uses an independent detecting circuit to detect the value of voltage so the accuracy is higher. This touch has high reliability, convenient operation, maintenance-free and addition ${ }^{(4)}$. It can be used in smart home, intelligent vehicle, modern office building and other occasions.

This paper will introduce a touch switch circuit based on PSoC. Section2 describe the operation principle of the circuit, the simulation and analysis be introduced in section 3 and last section is the conclusion of this paper.

\section{The Operation Principle of The Circuit}

\subsection{Structure}
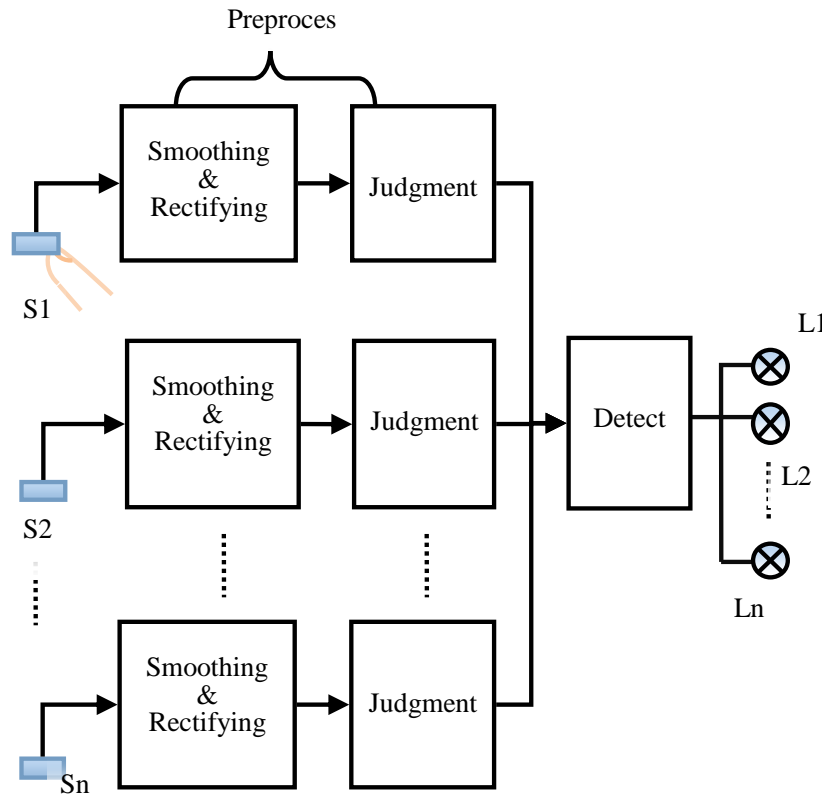

Fig.1. Structure of the touch switch circuit 
The touch switch circuit described in this paper can be divided into three parts:

(1) Metal film touch switch part

(2) Switching signal processing part

(3) Switching signal detection part

Fig. 1 shows the block diagram of the touch switch circuit. Each metal film touch switch has a process circuit to deal with the input signal. In order to distinguish the switches, a detection circuit is necessary. As it is known that if there is a lot of switches in the circuit, detection circuit should have the capability to judge which switch is pressed. In this circuit, the input voltage of detection circuit will be different depending on the different switch. Each part of the circuit will be discussed in detail below, here only introduce a switch as an example.

\subsection{Equivalent circuit of finger touch action}

The touch switch circuit is a kind of capacitance touch switch, touching the metal pieces is equal to add a small capacitance and a resistance into the circuit ${ }^{(5)}$. Fig. 2 shows the equivalent circuit of the finger touch part. The PWM wave which frequency is $100[\mathrm{~Hz}]$ and has $1 \mathrm{~ms}$ delay time simulates the action of finger contact. When finger touch the film is just like the output level of V2 is high level. The circuit is equal to access a $10[\mathrm{pF}]$ capacitor $\mathrm{C} 3$ and a $1[\mathrm{k} \Omega]$ resistor $\mathrm{R} 8$. If the finger leaves, just like the source V2 output low voltage and S2 is off. The terminal S2IN in connects to the input of U1A.

\subsection{Signal Processing Circuit}

The signal processing circuit is shown in Fig.3. There is a high frequency source V1 add to supply the input signal. Two equal resistors $\mathrm{R} 1, \mathrm{R} 2$ and capacitor $\mathrm{C} 1$ half the value of input square-wave voltage and no longer flat as there are some spikes in it. Because the capacitor C1 is very small, the capability of charge and discharge is very fast so the spikes appear. The switch terminal S2IN is connected into the noninverting input terminal of amplifier U1A, and then the change of the switch can be captured. The inverting input terminal of $\mathrm{U} 1 \mathrm{~A}$ is connected to the output terminal directly which makes the function similar to a buffer. The voltage of output of amplifier U1A is smoother than the input one. Then diode D1 and RC parallel circuit are combined into a rectifier circuit, the output average voltage is fluctuating around a constant value. The function of amplifier U1B is a voltage comparator. The voltage of noninverting input terminal is constant $2[\mathrm{~V}]$, which is the voltage at R5.
If the input voltage of inverting input terminal is above $2[\mathrm{~V}]$, the output voltage is almost $0[\mathrm{~V}]$, however, if less than or equal to $2[\mathrm{~V}]$, the output is a high level value. Controlled Switch S2 will connect only if the output voltage of $\mathrm{U} 1 \mathrm{~B}$ is above $1[\mathrm{~V}]$. Then the parallel resistors R6 and R7 will connect directly to $5 \mathrm{VDC}^{(6)}$. The other side of $\mathrm{R} 7$ is connected to $\mathrm{U} 1 \mathrm{C}$ inverting input just the line named U1C Input as shown in Fig.3.

\subsection{Detection Circuit}

In Fig. 4, the U1C Input terminal is connected with the feedback resistance R9. Once S2 is connected, the input voltage of amplifier U1C is generated. If R7 (in Fig.3) changes the value, the input voltage (current) is changed either. Therefore, when connecting multiple switches, different switches can be distinguished according to the different input values.

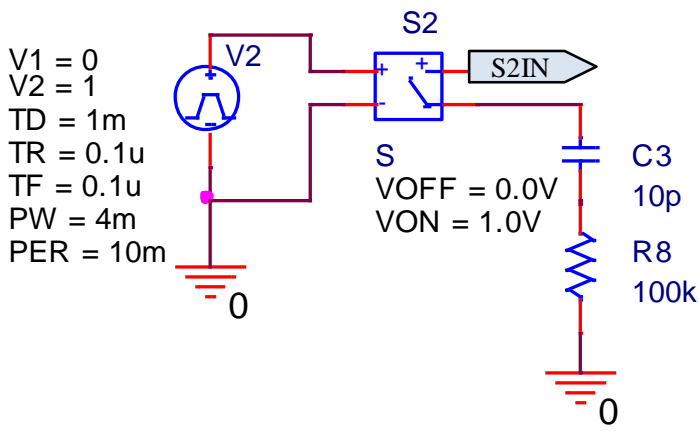

Fig.2. Equivalent circuit of finger touch action

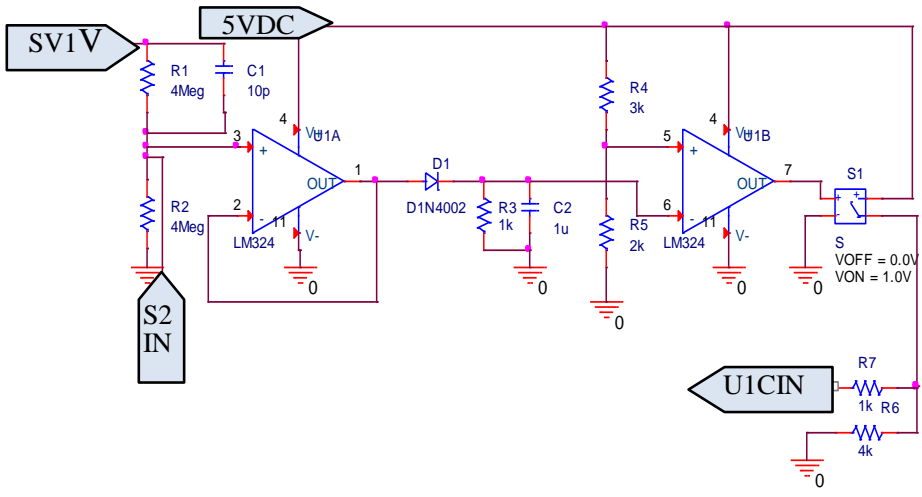

Fig.3. Signal processing circuit

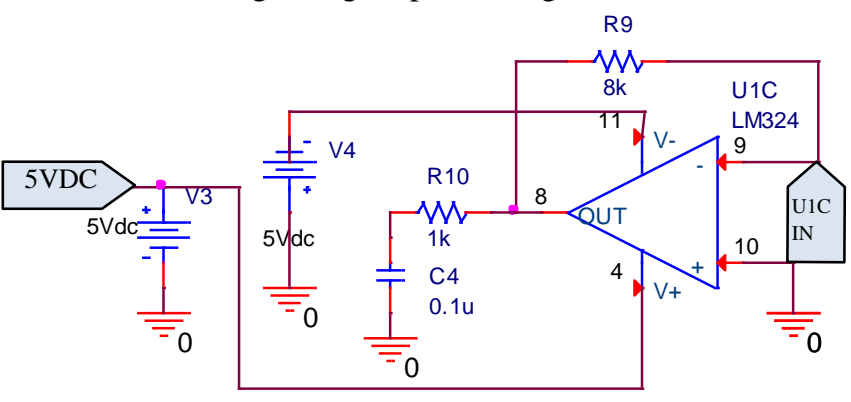

Fig.4. Switch detection circuit 


\section{Simulation and Discussion}

This paper uses PSPICE software from ORCAD Company to draw circuit diagrams and simulate ${ }^{(7)}$. It has powerful features of drawing schematic, circuit simulation, the graphics processing capability and component symbols design. This software has a graphical friendly interface, practical, good simulation effect, powerful and easy to operate.

Fig.5 shows the schematic of the circuit. The input pulse is a PWM wave with a frequency of $10[\mathrm{kHz}]$. After the processing circuit, the input voltage compares with a reference voltage then outputs a switching voltage. Only the switch is touched, the following detection circuit will work. Different combinations of the input resistances of the detection circuit will have different input voltage so that the measured voltage is not the same. If add same part of input circuit with different combinations of resistances, the whole circuit may become a multi-switching control circuit.

In order to watch the function of the circuit in the computer the simulation is required. The circuit can be simulated in the software and the voltage wave of any part in the circuit can be shown in the simulation window. First of all, we should set the simulation profile. The maximum step size of time has to be set at an appropriate size otherwise the simulation will generate an error about the voltage failed to converge. Sometimes the size is half of the minimum step size of signal generator, here the size is 10 [us].

We put one probe in the source signal generator V1 and another one in the input of the amplifier, and then run it. We can analysis the voltage wave as shown in Fig.6. The original power voltage is a square wave as the green line shows. After adding the equivalent circuit of finger touch switch, the input voltage of the amplifier U1A is like the red line shown in this figure which is divided into two parts.

In order to distinguish touch signal clearly the wave should be rectifying. As the values of touch part and untouched part have little difference around 2[V], a voltage comparator is used. The constant value of the non-intervening input terminal is $2 \mathrm{~V}$ just like the red line shown in Fig. 7. According the purple line, we can conclude that when the switch is touched, the input voltage of U1B is almost equal to $2[\mathrm{~V}]$ and the output voltage of U1B as the blue line is bigger than $1[\mathrm{~V}], \mathrm{S} 2$ is connected; the output voltage of S2 just like the yellow line is the DC voltage. If not, the output of U1B is almost $0[\mathrm{~V}]$ and S2 is off, there is no voltage on R6 and R7.

The detected circuit will work only when $\mathrm{S} 2$ is connected. The equivalent circuit shows in Fig.8. This is a negative feedback amplifier circuit. If the size value of R1 is changed, the input voltage is changed either. Output is a negative voltage.

If the value of $\mathrm{R} 1$ is $4[\mathrm{k} \Omega$ ], the input and output voltage waveform are like Fig.9, the value of input voltage which is green line is $1.678[\mathrm{~V}]$, the red line is output voltage which value is $-4.93[\mathrm{~V}]$; if the value of resistance is $1[\mathrm{k} \Omega]$, the input voltage is $3.893[\mathrm{~V}]$ as the blue line shown in Fig.10, the output is $-4.93[\mathrm{~V}]$ as purplish red line.

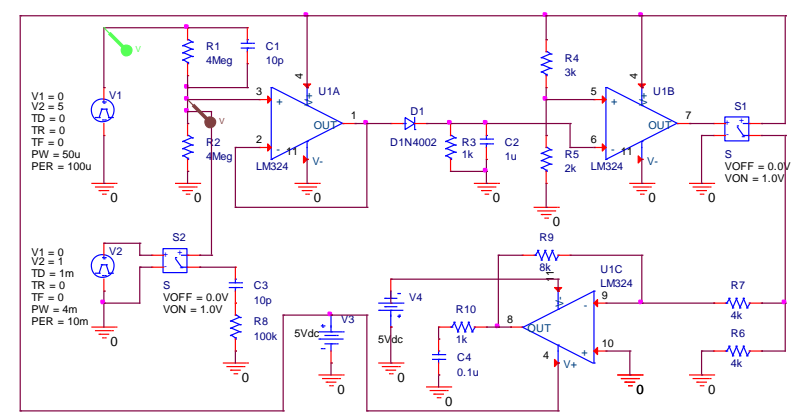

Fig.5. Schematic of the touch switch circuit

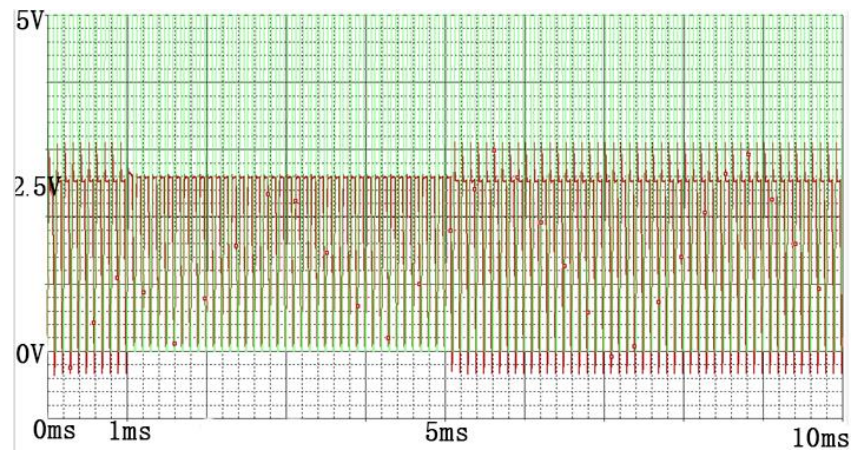

Fig.6. Input voltage waveform

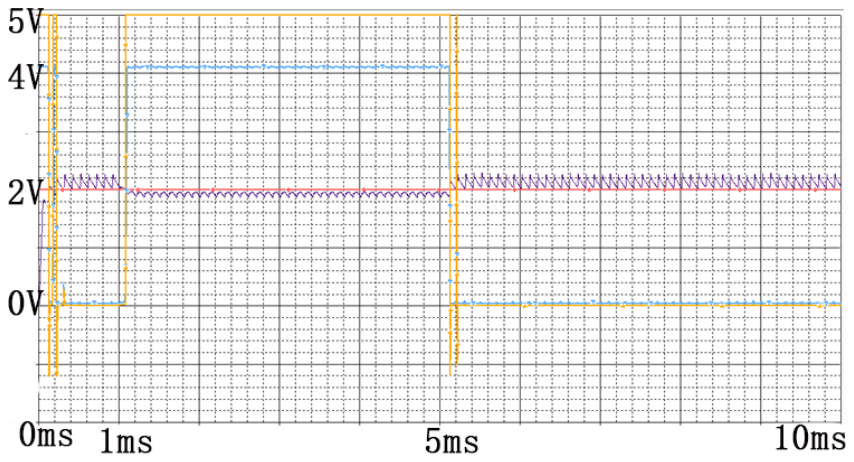

Fig.7. Waveform of U1B and S2 


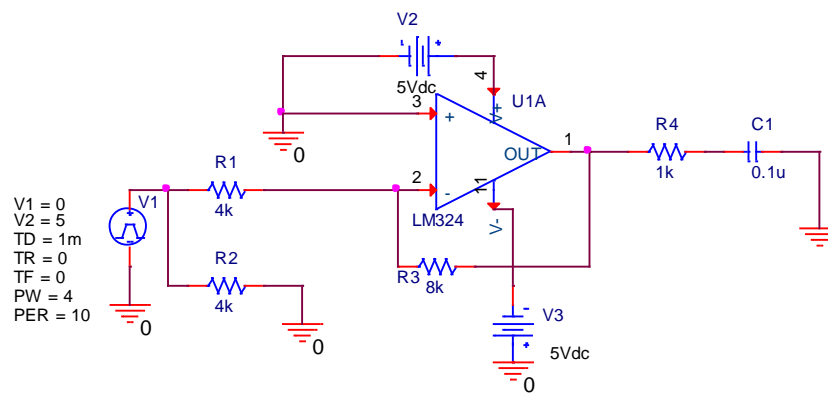

Fig.8. The equivalent circuit of detect parts

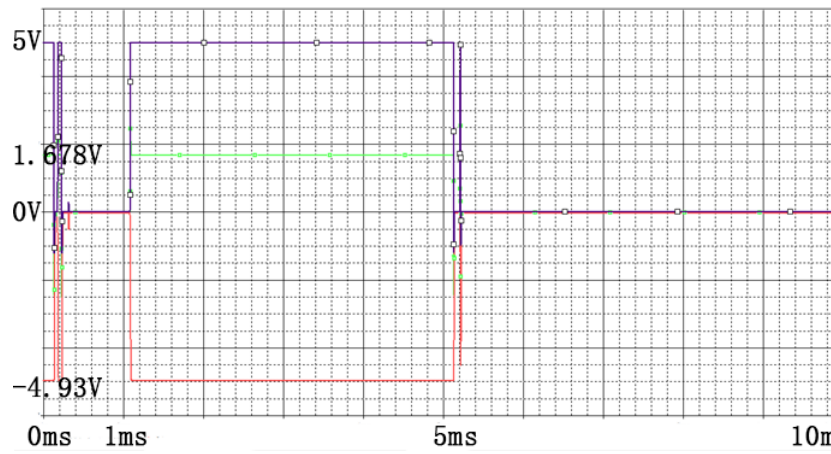

Fig.9. The voltage waveform of detect parts

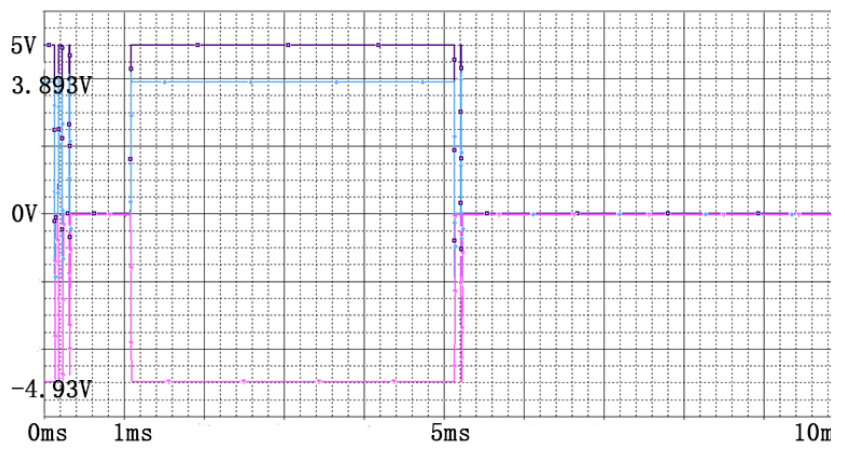

Fig.10. The voltage waveform of detect parts

\section{Conclusions}

After analysis every part of the circuit we can conclude that this touch switch circuit can achieve the function as a switch to control other equipments in ideal state. Besides, the shape of the switch can be changed because the switch is composed by the metal film. This switch is safety as finger touch part is separated with controlled part. According to the input voltage value of detection circuit, it can be easily distinguished if the switch is turn on or off. Different input resistances will produce different voltage value there for add switch is possible in this switch. The switch Combination of two resistors can realize nearly 100 possibilities, as long as increase a distinguish resistor, the number of increased switches can be added in exponential.

However, each switch has an individual processing circuit part so that if the number of switch increases may cause more power consumption.

In the future, the main work may focus on building up the physical circuit on circuit broad by using PSoC $^{(8)}$.Test it in different environment then increase more switches on this circuit and try to distinguish them. After that, robustness of the circuit has to be test to detect the stability of switch in different situation.

\section{References}

(1) Lingsheng Tian : "Analog switch type touch switch circuit", Journal of Electronics and Automation, Vol. 2, No. 1, pp. 40-41, 1998

(2) Chen Tang, Shiwen Li : "Simulation study of optimization design of touch switch", Journal of China Integrated Circuit, Vol. 81, No. 2, pp. 51-53, 2006

(3) Seiichi Serikawa, Lifeng Zhang : "Proposal of a touch panel switch with the function of bending and addition", $2^{\text {nd }}$ International Conference on ICICI-BME, pp. 8-13, 2011

(4) Liang Zhang, Mingfu Zhu : "Design of an inductive touch switch adaptive", Journal of Instrumentation equipment, Vol. 27, No. 3, pp. 27-29, 2007

(5) B.H. Shin, J.W. Yum and S. H. Kim : "Secure dual-chip contactless card with an integrated complementary touch switch", Journal of Microwave and optical technology letters, Vol. 55, No. 3, pp. 529-533, 2013

(6) Shibai Tong, Chengying Hua : "The basic of analog electronics", Higher education press, Vol. 2, pp. 171-193, 2001

(7) Yong Huang : "Simulation analysis and design of the circuit based on PSPICE", Journal of Hubei Institute of Education, Vol. 24, No. 2, pp. 35-37, 2007

(8) Huimin Lu, Seiichi Serikawa : "Design of freely configurable safety light curtain using hemispherical mirrors", IEEJ Transactions on Electrical and Electronic Engineering, Vol. 8, no.S1, pp. s110-s111, 2013 